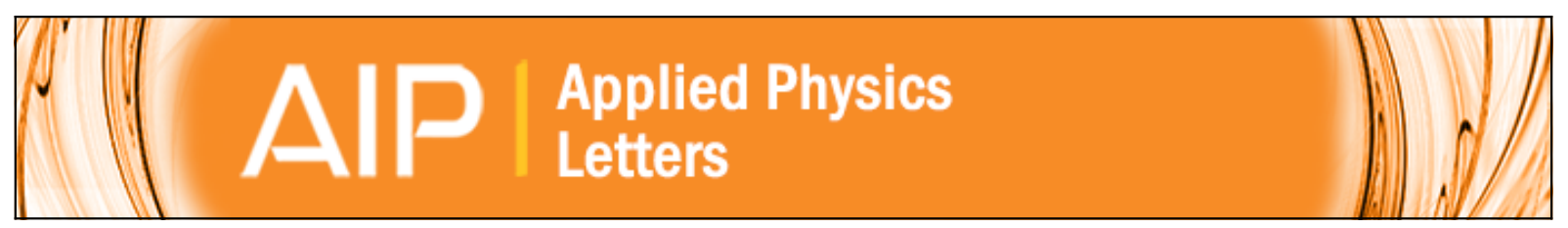

\title{
Imaging photocurrent collection losses in solar cells
}

Vito Huhn, Bart E. Pieters, Yael Augarten, Andreas Gerber, David Hinken, and Uwe Rau

Citation: Applied Physics Letters 109, 223502 (2016); doi: 10.1063/1.4971266

View online: http://dx.doi.org/10.1063/1.4971266

View Table of Contents: http://scitation.aip.org/content/aip/journal/apl/109/22?ver=pdfcov

Published by the AIP Publishing

\section{Articles you may be interested in}

Analysis of bulk and interface defects in hydrogenated amorphous silicon solar cells by Fourier transform photocurrent spectroscopy

J. Appl. Phys. 118, 184506 (2015); 10.1063/1.4935621

Photocurrent collection efficiency mapping of a silicon solar cell by a differential luminescence imaging technique Appl. Phys. Lett. 105, 163507 (2014); 10.1063/1.4898008

Origin of photocurrent generation and collection losses in large area organic solar cells Appl. Phys. Lett. 99, 093309 (2011); 10.1063/1.3637041

Photocurrent increase in $\mathrm{n}-\mathrm{i}-\mathrm{p}$ thin film silicon solar cells by guided mode excitation via grating coupler Appl. Phys. Lett. 96, 213508 (2010); 10.1063/1.3435481

Fourier-transform photocurrent spectroscopy of microcrystalline silicon for solar cells Appl. Phys. Lett. 80, 719 (2002); 10.1063/1.1446207

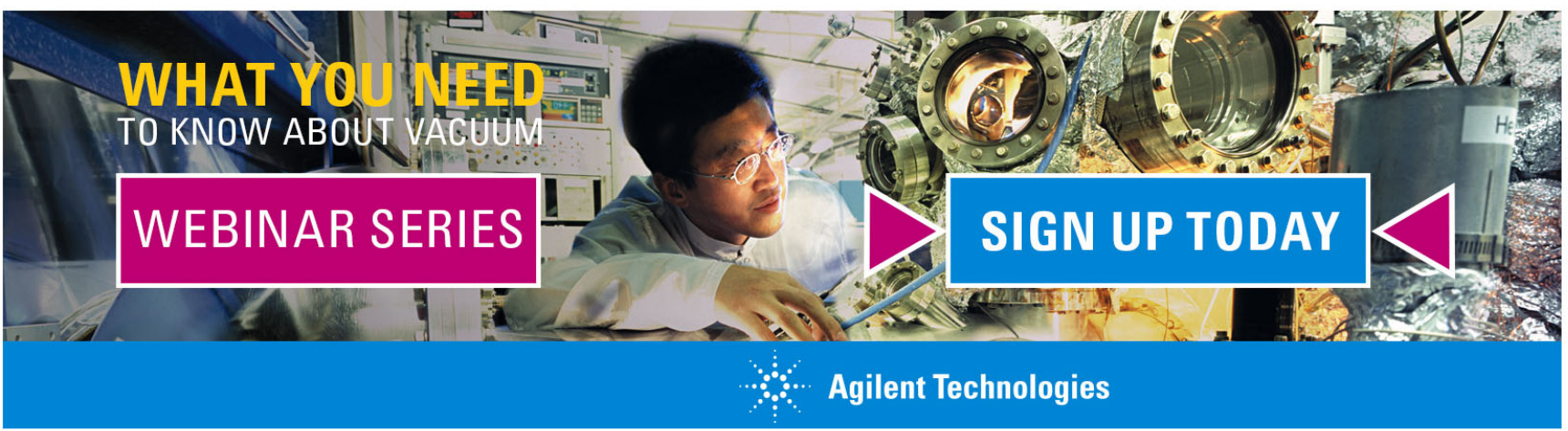




\title{
Imaging photocurrent collection losses in solar cells
}

\author{
Vito Huhn, ${ }^{1, a)}$ Bart E. Pieters, ${ }^{1}$ Yael Augarten, ${ }^{1}$ Andreas Gerber, ${ }^{1}$ David Hinken, ${ }^{2}$ \\ and Uwe Rau ${ }^{1}$ \\ ${ }^{1}$ IEK5-Photovoltaik, Forschungszentrum Jülich, 52425 Jülich, Germany \\ ${ }^{2}$ Institut für Solarenergieforschung GmbH Hameln, 31860 Emmerthal, Germany
}

(Received 10 August 2016; accepted 18 November 2016; published online 1 December 2016)

\begin{abstract}
A method is proposed that enables the imaging of the photocurrent collected by a solar cell under arbitrary operating conditions. The method uses a series of luminescence images under varying illumination to derive the total photocurrent collection efficiency at a given voltage bias. The resulting total photocurrent collection image directly relates to the difference between the dark and illuminated current-voltage characteristics of the cell. A crystalline silicon solar cell is used to test the method, and the images of the total photocurrent collection efficiency are used to quantify the influence of a crack on the total collected photocurrent of the solar cell. Published by AIP Publishing.

[http://dx.doi.org/10.1063/1.4971266]
\end{abstract}

During the recent years, luminescence imaging has become a standard tool to quantitatively analyze the performance of photovoltaic materials and devices. Some methods analyze the local properties of solar cells such as series resistance or dark saturation current. ${ }^{1-5}$ However, these evaluation methods rely on approximations and simplifications that are not universally valid in a spatially extended system of distributed diodes and resistors. ${ }^{6}$ Recently, a quantitative imaging method based on a linear reciprocity relationship for photocurrent collection was proposed. ${ }^{7}$ This method is universally valid and yields the differential local photocurrent collection efficiency $f_{\mathrm{pc}, \mathrm{loc}} \cdot{ }^{8-10}$ Hence, an image of $f_{\mathrm{pc}, \text { loc }}$ depicts the probability that a locally generated differential photocurrent arrives at the terminals of the solar cell at a given bias situation. Though being simple, general, and quantitative, the method only images a differential and not the integral situation.

The present paper extends the mapping of the differential photocurrent collection efficiency $f_{\mathrm{pc} \text {,loc }}$ to imaging of the total photocurrent collection efficiency $F_{\mathrm{pc}, \text { loc }}$. This quantity describes the fraction of the total locally generated photocurrent that contributes to the external current. Therefore, integration of the quantity $F_{\text {pc,loc }}$ over the cell area yields the difference between the dark and the illuminated current voltage characteristics, and $F_{\mathrm{pc} \text {,loc }}$ shows how much each location of the solar cell contributes to this difference.

The quantity $f_{\mathrm{pc}, \text { loc }}$ was defined by Wong and Green ${ }^{7}$ as

$$
\begin{aligned}
f_{\mathrm{pc}, \text { loc }}\left(\boldsymbol{r}, I_{\mathrm{ph}}, V_{\mathrm{ext}}\right) & :=\left.\frac{\delta I_{\text {coll,loc }}\left(\boldsymbol{r}, I_{\mathrm{ph}}(\boldsymbol{r}), V_{\mathrm{ext}}\right)}{\delta I_{\mathrm{ph}}(\boldsymbol{r})}\right|_{\delta V_{\mathrm{ext}}=0} \\
& =\left.\frac{\delta V_{\mathrm{j}}\left(\boldsymbol{r}, I_{\mathrm{ph}}(\boldsymbol{r}), V_{\mathrm{ext}}\right)}{\delta V_{\mathrm{ext}}}\right|_{\delta I_{\mathrm{ph}}=0},
\end{aligned}
$$

where $I_{\text {coll,loc }}$ describes the externally collected current and $I_{\mathrm{ph}}$ describes the generated photocurrent at position $\boldsymbol{r}$. The quantity $f_{\mathrm{pc}, \text { loc }}$ is therefore a differential measure for the

\footnotetext{
${ }^{\text {a) }}$ Author to whom correspondence should be addressed. Electronic mail: v.huhn@fz-juelich.de
}

fraction of the locally generated photocurrent that contributes to the current at the terminals of the device. Equation (1) also shows that $f_{\mathrm{pc}, \text { loc }}$ is equal to the derivative of the local junction voltage $V_{\mathrm{j}}$ with respect to the external voltage $V_{\text {ext }}$ under constant illumination. The local junction voltage $V_{\mathrm{j}}$ is accessible from luminescence images and the reciprocity relation ${ }^{11}$ by

$$
\begin{aligned}
\Phi_{\mathrm{EM}-\mathrm{PL}}\left(E, \boldsymbol{r}, V_{\mathrm{ext}}, \Phi_{\mathrm{exc}}\right)= & \Phi_{\mathrm{SC}}\left(E, \boldsymbol{r}, \Phi_{\mathrm{exc}}\right)+Q_{\mathrm{e}}(E, \boldsymbol{r}) \Phi_{\mathrm{bb}}(E) \\
& \times\left[\exp \left(\frac{V_{\mathrm{j}}\left(\boldsymbol{r}, I_{\mathrm{ph}}(\boldsymbol{r}), V_{\mathrm{ext}}\right)}{V_{\mathrm{th}}}\right)-1\right] .
\end{aligned}
$$

In Eq. (2), it is shown how the luminescence intensity $\Phi_{\mathrm{EM}-\mathrm{PL}}$ depends on the junction voltage $V_{\mathrm{j}}$. The quantity $\Phi_{\mathrm{bb}}$ is the black body spectrum, $Q_{\mathrm{e}}$ is the external quantum efficiency, and $\Phi_{\mathrm{SC}}$ is the luminescence intensity under short circuit conditions, which is voltage independent but is influenced by the illumination intensity $\Phi_{\text {exc }}$. The energy of the emitted photons is described by $E$ and the thermal voltage is described by $V_{\text {th }}$. The differential photocurrent collection efficiency $f_{\mathrm{pc}, \text { loc }}$ is determined by taking luminescence images at varying external voltages and determining $\delta V_{\mathrm{j}}$ using Eq. (2). The details of the measurement and calculation procedure have been published previously. 8,9

For our purpose, we have to calculate the total externally collected photocurrent $I_{\text {coll,loc }}$ resulting from the generated photocurrent at position $\boldsymbol{r}$ according to

$$
I_{\mathrm{coll}, \mathrm{loc}}\left(V_{\mathrm{ext}}, I_{\mathrm{ph}}, \boldsymbol{r}\right)=\int_{0}^{I_{\mathrm{ph}}} f_{\mathrm{pc}, \mathrm{loc}}\left(\boldsymbol{r}, I_{\mathrm{ph}}^{\prime}, V_{\mathrm{ext}}\right) d I_{\mathrm{ph}}^{\prime} .
$$

Equation (3) states that we need $f_{\mathrm{pc} \text {,loc }}$ images at different illumination intensities and the locally generated photocurrent $I_{\mathrm{ph}}$. For the following, we assume that the quantum efficiency of the analyzed device is position independent and that $f_{\mathrm{pc}, \mathrm{loc}}=1$ at $V_{\mathrm{ext}}=0$, everywhere in the device. Following these assumptions, $I_{\mathrm{ph}}$ can be determined from the short circuit current $I_{\mathrm{sc}}$, as the short circuit current density $J_{\mathrm{sc}}$ is then equal to the locally generated photocurrent density $J_{\mathrm{ph}}$. The desired quantity $F_{\mathrm{pc} \text {,loc }}$ is obtained from Eq. (3) simply by 
dividing through the locally generated photocurrent. After additionally transferring currents to current densities and using $J_{\mathrm{ph}}=J_{\mathrm{sc}}$, Eq. (3) reads

$$
F_{\mathrm{pc}, \mathrm{loc}}\left(\boldsymbol{r}, J_{\mathrm{sc}}, V_{\mathrm{ext}}\right)=\frac{1}{J_{\mathrm{sc}}} \int_{0}^{J_{\mathrm{sc}}} f_{\mathrm{pc}, \mathrm{loc}}\left(\boldsymbol{r}, J_{\mathrm{sc}}^{\prime}, V_{\mathrm{ext}}\right) d J_{\mathrm{sc}}^{\prime} .
$$

To illustrate the meaning of the $f_{\mathrm{pc}, \text { loc }}$ and $F_{\mathrm{pc}, \text { loc }}$ images, we discuss in the following how these local properties of a solar cell relate to a global property that is determined without the use of imaging techniques. The global externally collected photocurrent is defined via

$$
I_{\mathrm{coll}, \mathrm{glob}}\left(V_{\mathrm{ext}}, I_{\mathrm{sc}}\right)=I\left(V_{\mathrm{ext}}, I_{\mathrm{sc}}\right)-I\left(V_{\mathrm{ext}}, I_{\mathrm{sc}}=0\right),
$$

i.e., as the difference between a dark and illuminated current-voltage curve at the same $V_{\text {ext }}$. Furthermore, we define a global differential photocurrent collection efficiency $f_{\mathrm{pc}, \text { glob }}$ as the derivative of $I_{\text {coll,glob }}$ with respect to the short circuit current. Accordingly, the integral quantity $F_{\mathrm{pc}, \text { glob }}$ equals $I_{\mathrm{coll}, \mathrm{glob}} / I_{\mathrm{sc}}$ and we have

$$
\begin{aligned}
f_{\mathrm{pc}, \mathrm{glob}}\left(V_{\mathrm{ext}}, I_{\mathrm{sc}}\right) & =\frac{d I_{\mathrm{coll}, \mathrm{glob}}\left(V_{\mathrm{ext}}, I_{\mathrm{sc}}\right)}{d I_{\mathrm{sc}}} \\
& =\frac{1}{A} \iint f_{\mathrm{pc}, \mathrm{loc}}\left(\boldsymbol{r}, I_{\mathrm{sc}}, V_{\mathrm{ext}}\right) d A,
\end{aligned}
$$

as well as

$$
\begin{aligned}
F_{\mathrm{pc}, \mathrm{glob}}\left(V_{\mathrm{ext}}, I_{\mathrm{sc}}\right) & =\frac{I_{\mathrm{coll}, \mathrm{glob}}\left(V_{\mathrm{ext}}, I_{\mathrm{sc}}\right)}{I_{\mathrm{sc}}} \\
& =\frac{1}{A} \iint F_{\mathrm{pc}, \mathrm{loc}}\left(\boldsymbol{r}, I_{\mathrm{sc}}, V_{\mathrm{ext}}\right) d A .
\end{aligned}
$$

In Eqs. (6) and (7), $A$ denotes the active area of the sample. The above relations are useful to verify the assumptions and the theory behind the method, as they provide a simple way to relate the imaging results [right terms of Eqs. (6) and (7)] to the properties of the device that can be measured independently from the current-voltage curves [middle terms of Eqs. (6) and (7)]. Note that Eq. (6) was already used by Delamarre $e t$ al. to verify the differential photocurrent collection efficiency images. ${ }^{12}$

The method was tested with a crystalline silicon solar cell. A standard PL imaging system was used. The illumination source was a $90 \mathrm{~W}, 808 \mathrm{~nm}$ diode laser that was widened with a beam homogenizer to an overall homogeneity of $\pm 5 \%$ on a $20 \times 20 \mathrm{~cm}^{2}$ area. The maximum possible illumination intensity is therefore $0.225 \mathrm{~W} / \mathrm{cm}^{2}$. The illumination spectrum plays only a minor role for photocurrent collection imaging. It influences the illumination intensity dependence of the operating point of the solar cell, i.e., current and voltage, but as long as the operating point is the same, the photocurrent collection images are independent of the illuminating spectrum. The images were taken with a Princeton Instruments Nirvana640 InGaAs camera $(640 \times 512$ pixels $)$ cooled to $-80^{\circ} \mathrm{C}$. A $12.5 \times 12.5 \mathrm{~cm}^{2} \mathrm{c}$-Si solar cell was placed on a sample holder with a fixed temperature and electrically connected. Temperature control is necessary, as the sample must remain at a fixed temperature $\left(25^{\circ} \mathrm{C}\right)$ even if the illumination changes. Luminescence images were taken under different illumination and voltage conditions. The voltage point $V_{\text {ext }}$ was varied from $0.45 \mathrm{~V}$ to $0.6 \mathrm{~V}$ (from maximum power point to open circuit voltage) in $0.05 \mathrm{~V}$ steps at each illumination intensity. The illumination intensity was varied between 0 and $0.115 \mathrm{~W} / \mathrm{cm}^{2}$ in 12 steps. This corresponds to a highest short circuit current density $J_{\mathrm{sc}}$ of $32 \mathrm{~mA} / \mathrm{cm}^{2}$, with $J_{\mathrm{sc}}=32.7 \mathrm{~mA} / \mathrm{cm}^{2}$ for the solar cell under simulated AM1.5 illumination. Figure 1 shows a luminescence image taken at $0.6 \mathrm{~V}$ under maximum illumination intensity. From the figure, we clearly see several cracks and defects in the sample.

To determine $f_{\mathrm{pc}, \text { loc }}$ using the log-based method ${ }^{9,13}$ for each voltage point, we used images taken at $0.01 \mathrm{~V}$ above and below the voltage point of interest and one image with the external voltage set to zero. The results for the $f_{\mathrm{pc}, \text { loc }}$ images calculated at the maximum illumination intensity are shown in Fig. 2. These images can be used to give a first quantification of the influence the defects have on the photocurrent collection. The cracks and high series resistance regions clearly reduce the ability of the solar cell to collect generated photocurrent. It can also be seen that $f_{\mathrm{pc}, \text { loc }}$ decreases in general with the increasing voltage. This decrease results from the decrease in the differential diode resistance towards higher voltages compared to the series resistances within the device. ${ }^{9}$

Following the imaging of $f_{\mathrm{pc}, \text { loc }}$, we determine the total local photocurrent collection efficiency $F_{\mathrm{pc} \text {,loc }}$. This quantity is calculated by integration of $f_{\mathrm{pc} \text {,loc }}$ over the different illumination intensities, as described by Eq. (4). The calculated $F_{\mathrm{pc}, \mathrm{loc}}$ images are shown in Fig. 3. Due to the larger $f_{\mathrm{pc}, \text { loc }}$ at lower illumination intensities, $F_{\mathrm{pc}, \text { loc }}$ is always larger than $f_{\mathrm{pc}, \text { loc }}$ for the same values for external voltage and illumination. At $V_{\text {ext }}=0.45 \mathrm{~V}$ (close to the maximum power point), the defected region [marked by the green frame in Fig. 3(a), area $\left.A_{\mathrm{g}}=5.3 \mathrm{~cm}^{2}\right]$ has an average $F_{\mathrm{pc}, \text { loc }}$ of 0.52 . Multiplying area and average $F_{\mathrm{pc} \text { loc }}$ with the short circuit current density of $32 \mathrm{~mA} / \mathrm{cm}^{2}$ yields a photocurrent of $88 \mathrm{~mA}$ collected from that region. In contrast, a region with the same size and form but without the crack [marked by the red frame in Fig. 3(a)] has a mean $F_{\mathrm{pc}, \mathrm{loc}}$ of 0.74 and therefore yields a collected photocurrent of $126 \mathrm{~mA}$. Thus, we lose $38 \mathrm{~mA}$ due to the crack. As the total amount of photocurrent that is collected by the cell at $0.45 \mathrm{~V}$ under these illumination conditions is $3.01 \mathrm{~A}$, the

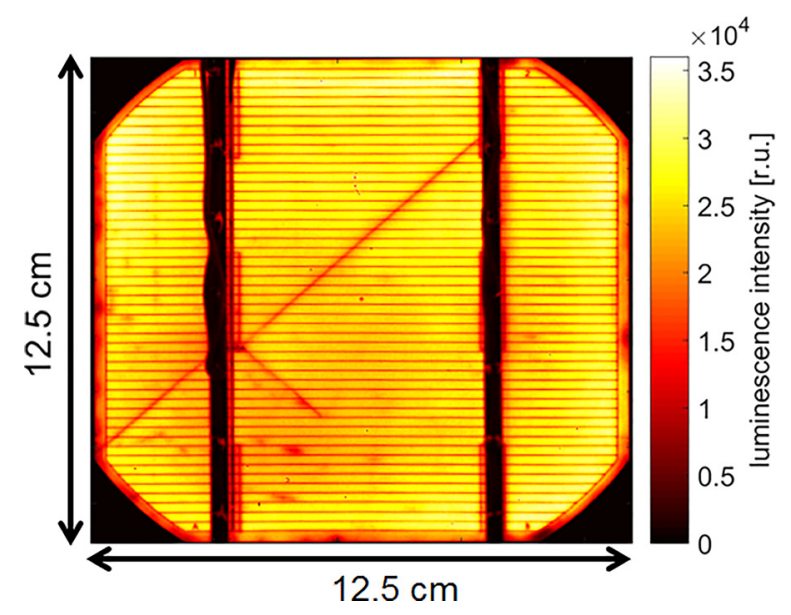

FIG. 1. Luminescence image taken at $0.6 \mathrm{~V}$ and an illumination intensity of $0.115 \mathrm{~W} / \mathrm{cm}^{2}$ (corresponding to a short circuit current density $J_{\mathrm{sc}}=32 \mathrm{~mA} / \mathrm{cm}^{2}$ ). 

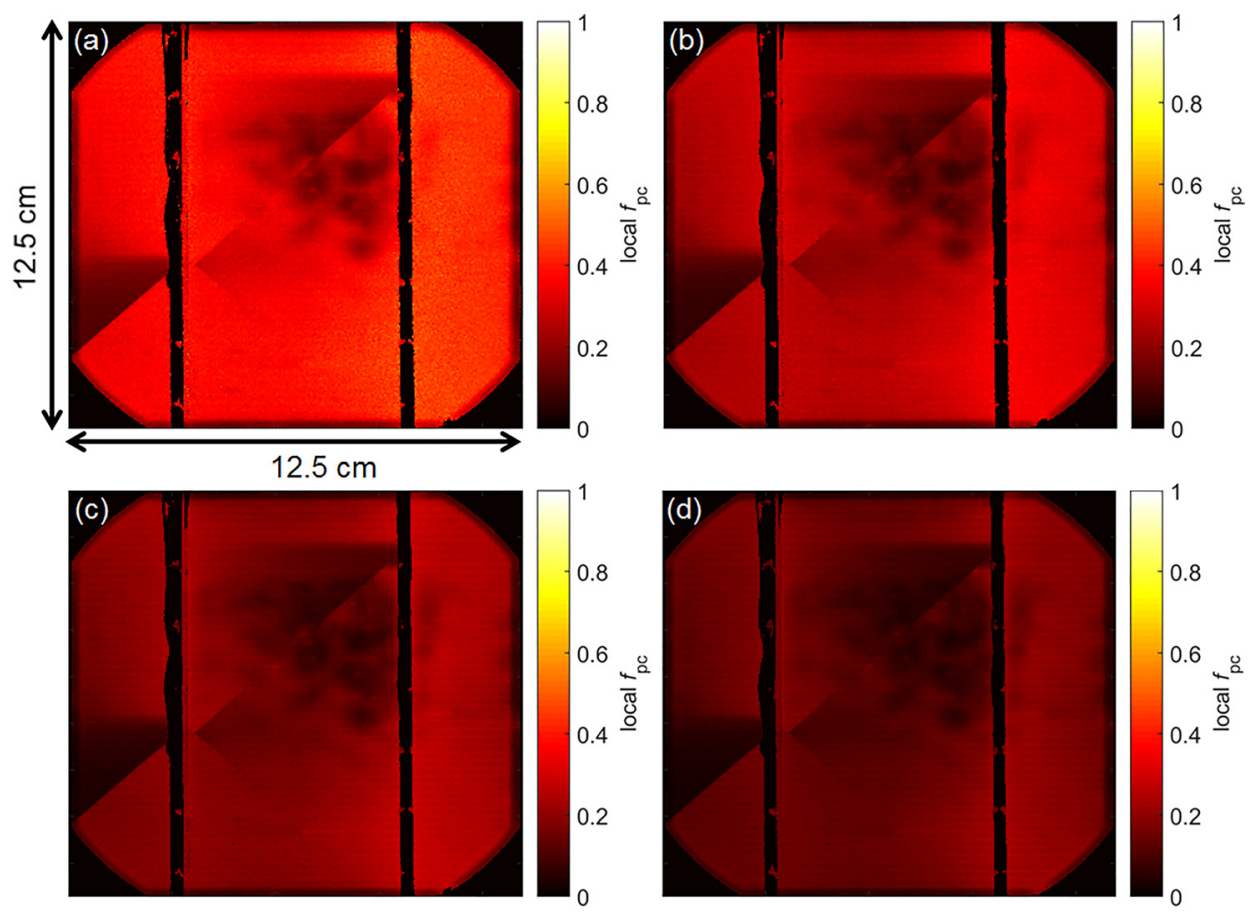

FIG. 2. Differential photocurrent collection efficiency images determined for the operating points (a) $0.45 \mathrm{~V}$, (b) $0.5 \mathrm{~V}$, (c) $0.55 \mathrm{~V}$, and (d) $0.6 \mathrm{~V}$ at an illumination intensity of $0.115 \mathrm{~W} / \mathrm{cm}^{2}$.

amount of collected photocurrent would therefore increase by roughly $1.3 \%$ if the crack would not be present.

Finally, Figure 4 compares the quantities $f_{\mathrm{pc}, \text { glob }}$ and $F_{\text {pc,glob }}$ determined from the collection efficiency images with the values of $f_{\mathrm{pc}, \mathrm{glob}}$ and $F_{\mathrm{pc} \text {,glob }}$ determined from the electrical measurements in order to quantitatively validate our method. For the electrical measurements, the current changes $d I_{\text {coll,glob }}$ resulting from illumination changes at different fixed $V_{\text {ext }}$ are measured and we use $f_{\mathrm{pc}, \mathrm{glob}}=d I_{\text {coll,glob }}\left(V_{\text {ext }}, I_{\mathrm{sc}}\right) / d I_{\mathrm{sc}}$ (depicted as blue symbols in Fig. 4) where $d I_{\mathrm{sc}}$ denotes the short circuit current change. The values for $f_{\mathrm{pc}, \text { glob }}$ determined via averaging of the collection efficiency images are shown in red. The quantities were measured for different voltage points and at different illumination conditions (given at the $\mathrm{x}$-axis by the short circuit current density of the device). The measurements were repeated five times and the standard deviation was used to estimate the errors. The errors of the electrical measurements are larger especially at higher voltages, as the temperature variations of the sample resulting from the illumination changes play a larger role there. This effect does not play a role for the imaging results as they are measured under constant illumination conditions. Since in both cases small differences of large values are measured, initially, small relative errors lead to larger relative errors of $f_{\mathrm{pc}}$. The inset in Fig. 4 shows the resulting $F_{\mathrm{pc}, \text { glob }}$ values for the different external voltages at the maximum illumination intensity. Again, the results from the electrical measurements and those calculated from the luminescence

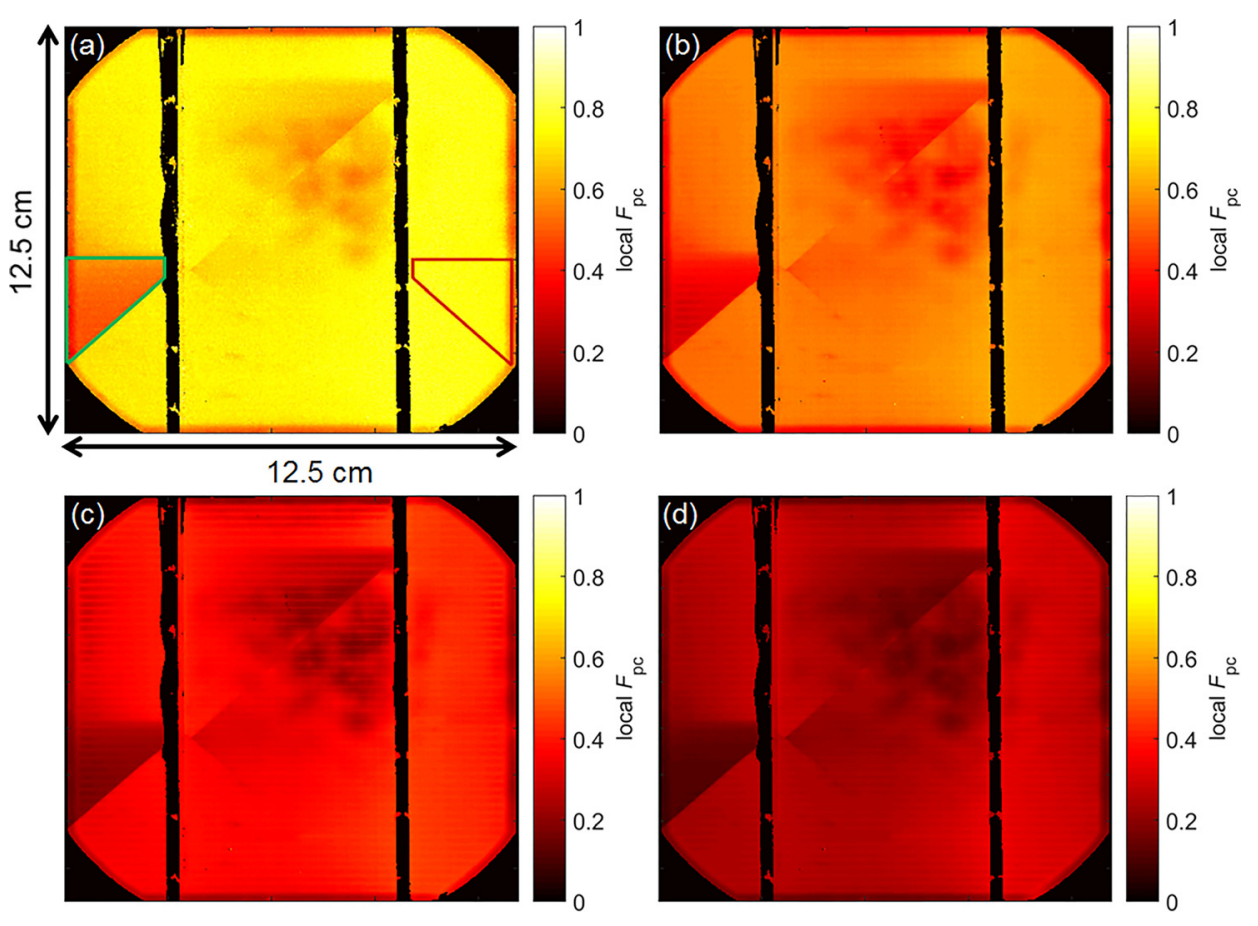

FIG. 3. Total photocurrent collection efficiency images determined for the operating points (a) $0.45 \mathrm{~V}$, (b) $0.5 \mathrm{~V}$, (c) $0.55 \mathrm{~V}$, and (d) $0.6 \mathrm{~V}$ for an illumination intensity of $0.115 \mathrm{~W} / \mathrm{cm}^{2}$. The green framed region in (a) was estimated to be influenced by a crack and analyzed further. It was found by a comparison with the red framed area in (a) that the collected photocurrent at $V_{\text {ext }}=0.45 \mathrm{~V}$ was reduced by approximately $1.3 \%$ due to the reduction of $F_{\text {pc,loc }}$ in the green framed area. 


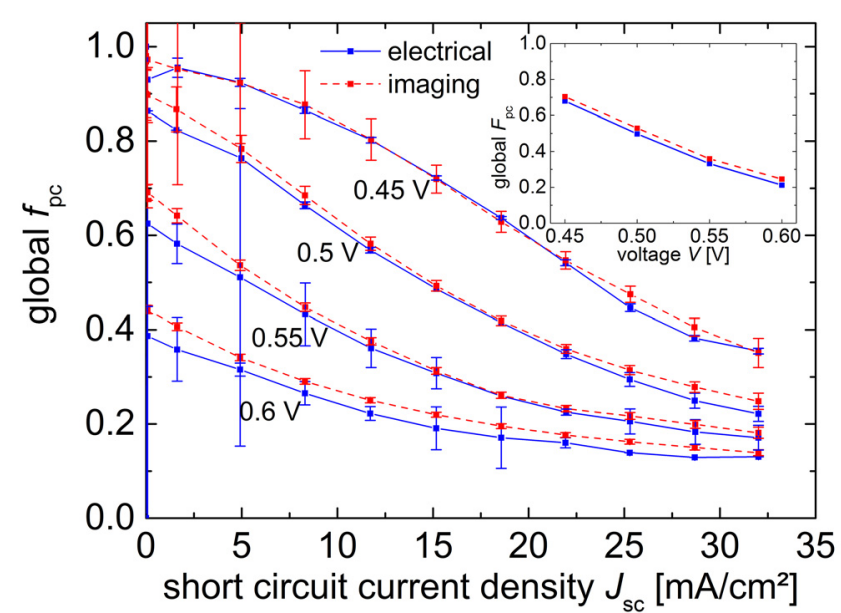

FIG. 4. Global differential photocurrent collection efficiency determined for different voltage points and illumination conditions via electrical measurements and imaging. The inset shows the global total photocurrent collection efficiency calculated at different external voltages for the maximum illumination condition.

images are in good agreement, validating the predictions of Eqs. (6) and (7).

In summary, we have introduced a luminescence imaging-based method for the spatially resolved determination of the photocurrent collection efficiency. The method is strictly based on a generally valid network theorem. ${ }^{7}$ The only additional assumption is that the locally generated photocurrent $I_{\mathrm{ph}}$ is position independent. This assumption is uncritical because variations of $I_{\mathrm{ph}}$, if any, are usually in the range of few $\%$ and introduce only a similar error in the results. For the previous differential method, ${ }^{8,10}$ three luminescence images are needed for each voltage point to determine the differential collection efficiency $f_{\mathrm{pc}, \text { loc }}$. This method is suitable for a quick, systematic monitoring of defects in solar cells. However, as a differential quantity, $f_{\mathrm{pc} \text {.loc }}$ is of limited use and, because $f_{\mathrm{pc}, \text { loc }} \leq F_{\mathrm{pc} \text {,loc }}$, it overestimates the consequences of defects. The integral method presented here needs measurements at several illumination intensities between zero and the desired operating point (in the present work, we used 36 images per operating point). However, the measurement procedure itself can be easily automated and it is suitable for in-depth analysis of defects as well as for the verification of grid and contact designs of solar cells.

This work has been supported by the German Ministry for Economy and Energy within the project "OptiCIGS" (FKZ 0325724A).

${ }^{1}$ T. Fuyuki, H. Kondo, T. Yamazaki, Y. Takahashi, and Y. Uraoka, Appl. Phys. Lett. 86, 262108 (2005).

${ }^{2}$ T. Trupke, R. A. Bardos, M. C. Schubert, and W. Warta, Appl. Phys. Lett. 89, 044107 (2006).

${ }^{3} \mathrm{~K}$. Bothe and D. Hinken, in Semiconductors and Semimetals Vol. 89, Advances in Photovoltaics Vol. 2, edited by G. P. Willeke and E. R. Weber (Academic Press, Burlington, 2013), p. 259.

${ }^{4}$ M. Glatthaar, J. Haunschild, M. Kasemann, J. Giesecke, W. Warta, and S. Rein, Phys. Status Solidi RRL 4, 13 (2010).

${ }^{5}$ D. Hinken, K. Ramspeck, K. Bothe, B. Fischer, and R. Brendel, Appl. Phys. Lett. 91, 182104 (2007).

${ }^{6}$ O. Breitenstein, J. Bauer, D. Hinken, and K. Bothe, Sol. Energy Mater. Sol. Cells 137, 50 (2015).

${ }^{7}$ J. Wong and M. A. Green, Phys. Rev. B 85, 235205 (2012).

${ }^{8}$ U. Rau, V. Huhn, L. Stoicescu, M. Schneemann, Y. Augarten, A. Gerber, and B. E. Pieters, Appl. Phys. Lett. 105, 163507 (2014).

${ }^{9}$ V. Huhn, A. Gerber, Y. Augarten, B. E. Pieters, and U. Rau, J. Appl. Phys. 119, 095704 (2016).

${ }^{10}$ J. Wong, R. Sridharan, Y. C. Wang, and T. Mueller, in IEEE 40th Photovoltaic Specialist Conference (PVSC), Denver, CO, 2014, p. 0975.

${ }^{11}$ U. Rau, IEEE J. Photovoltaics 2, 169 (2012).

${ }^{12}$ A. Delamarre, L. Lombez, K. Watanabe, M. Sugiyama, Y. Nakano, and J. F. Guillemoles, IEEE J. Photovoltaics 6, 528 (2016).

${ }^{13}$ J. Wong, IEEE Trans. Electron Devices 60, 917 (2013). 